
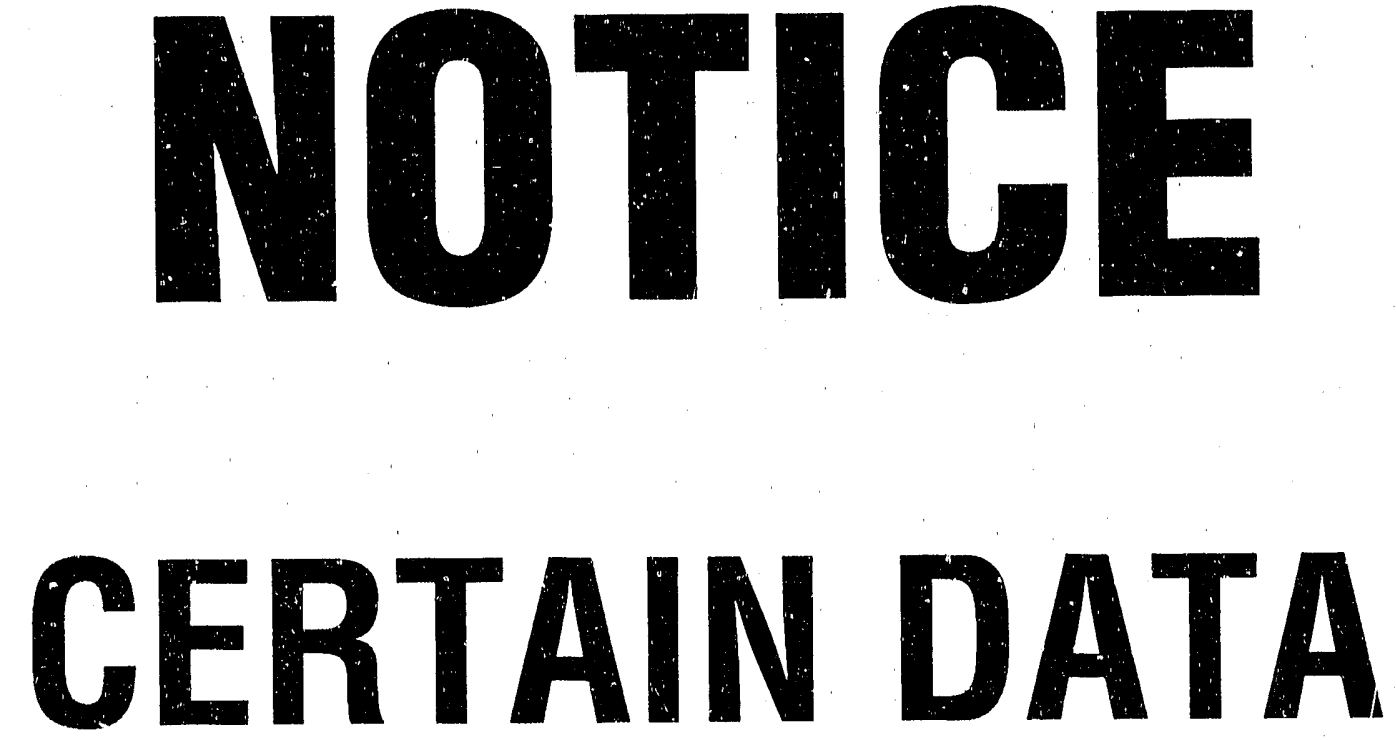

CONTAINED IN THIS
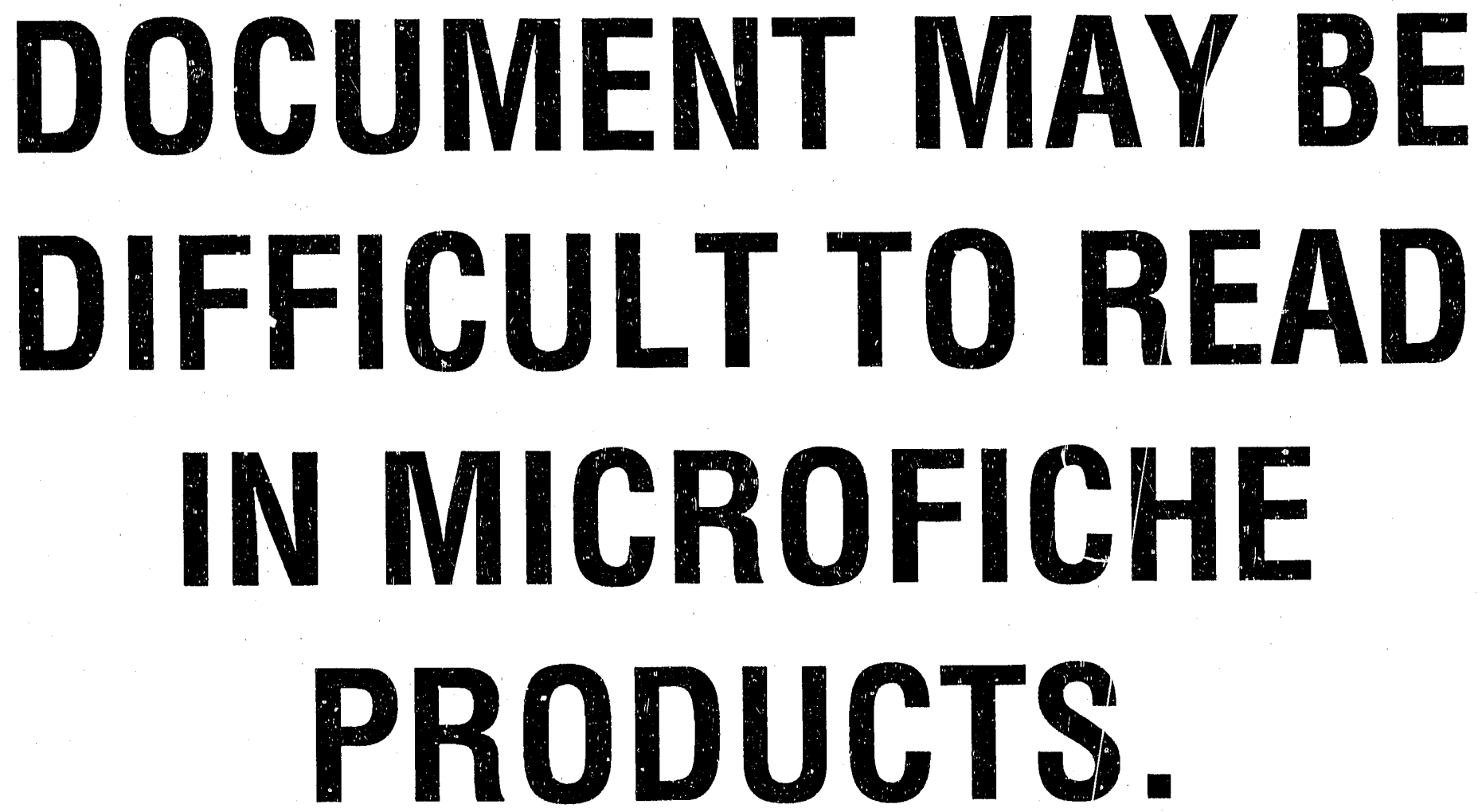
May 24, 1990

\author{
Annual Report \\ Covering the Period June 1, 1989 to May 31, 1990
}

\title{
COLLISIONAL PROCESSES OF INTEREST \\ IN MFE PLASMA RESEARCH
}

DOE/ER/ $53175--5$

DE9 1000598
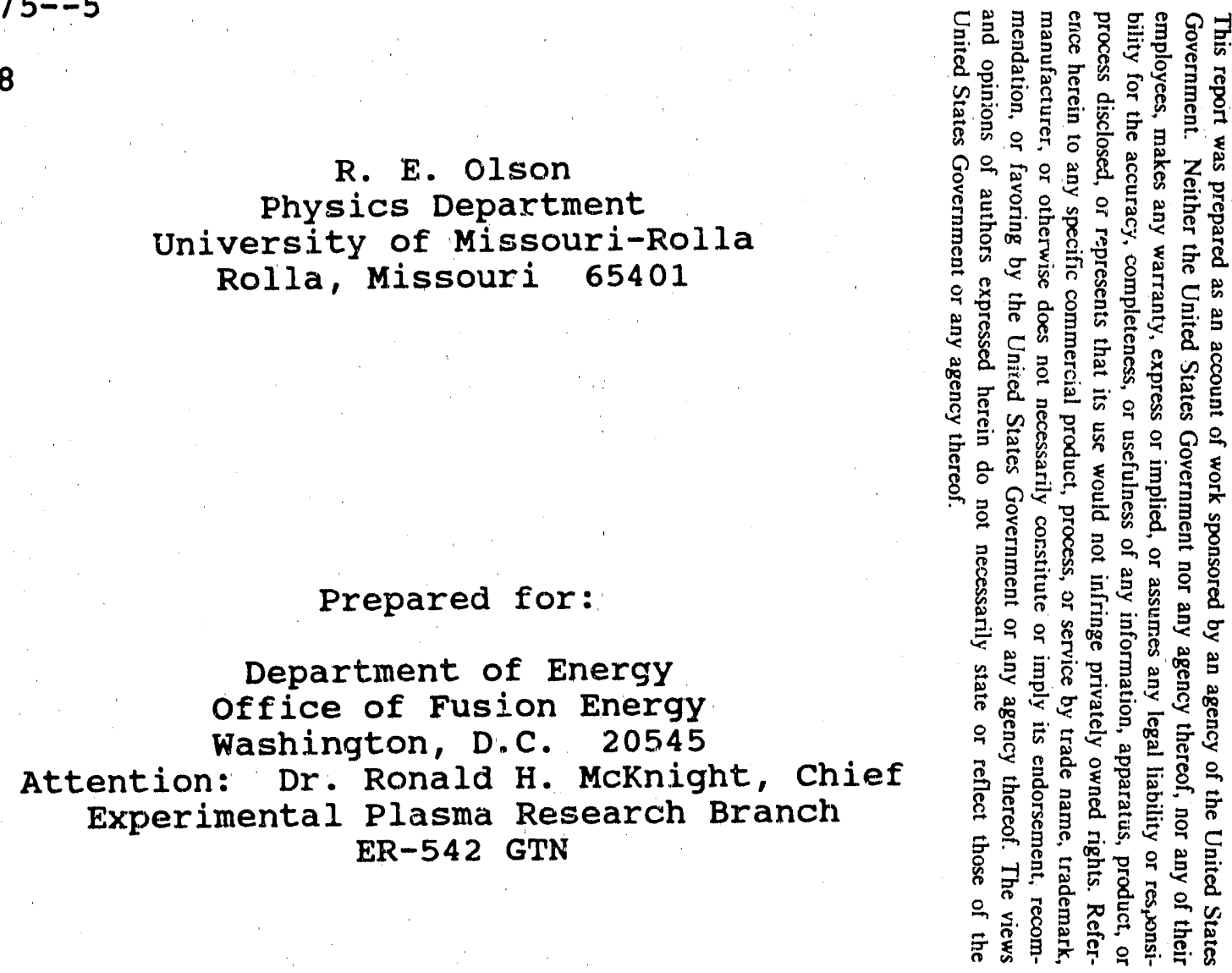

DOE Contract DE-FG02-84ER53175 


\begin{abstract}
Research on this grant is devoted to the calculation of heavy particle collision cross sections needed for diagnostic studies of magnetic fusion plasmas. This work requires the development and testing of new theoretical methods, with the implementation of benchmarked techniques to collisions pertinent to fusion reactors. Within the last context, we have provided charge-exchange-recombination cross sections to specific n, l-levels for diagnostic studies on TFTR and for a major compilation for IAEA. We have also completed a cross section study related to the planned neutral beam current drive for ITER. In addition, calculations were completed to assess the use of He neutral atom angular scattering measurements for JT-60. Also, new theoretical methods have been developed to more accurately calculate cross sections involving either $\mathrm{He}$ or $\mathrm{H}_{2}$ targets and partially stripped multiply-charged ions.
\end{abstract}




\section{PROGRESS REPORT}

our research accomplishments for the period June 1 , 1989 to May 31, 1990 are reported below. The papers published on the various topics during the last year are listed at the end of each subsection.

\section{A. IMPURTTY ION CHARGE-EXCHANGE-RECOKBINATION}

We completed a large series of calculations to determine the $n, 1$ product distributions for collisions of

$$
c^{6+}+H \rightarrow c^{5+}(n, 1)+H^{+}
$$

and

$$
\mathrm{O}^{8+}+\mathrm{H} \rightarrow \mathrm{O}^{7+}(\mathrm{n}, 1)+\mathrm{H}^{+}
$$

at energies from $40 \mathrm{keV} / \mathrm{u}$ to $140 \mathrm{keV} / \mathrm{u}$. This work was published in tabulation form by an IAEA sponsored publication in Physica scripta.

A complementary series of calculations for the $n, l$ charge-exchange-recombination cross sections were made for the $\mathrm{He}^{++}+\mathrm{H}$ and $\mathrm{N}^{7+}+\mathrm{H}$ reactions. These data, and the $\mathrm{C}^{6+}$ and $0^{8+}$ results, were transmitted via floopy disk to Ron Phaneuf at the Oak Ridge and Ranko Janev at IAEA Data Centers for inclusion in their fusion energy cross section data bases.

Prycica Scripe. Vod. Tat, $11-x$, 1 ses.

$n, I$ Distributions for Electron-Capture from $H(1 s)$ by $\mathrm{C}^{6+}$ and $\mathrm{O}^{\mathrm{at}}$

\section{R. E. Orson and D. R. Schulle}

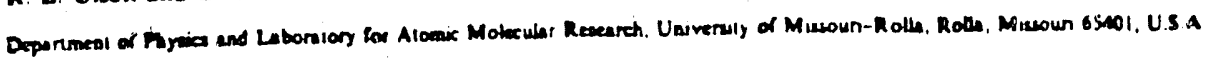

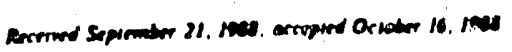

Atrian

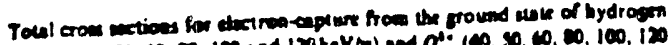

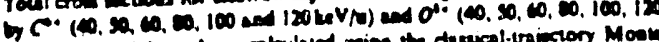

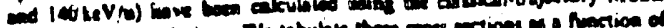

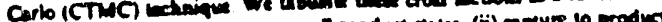

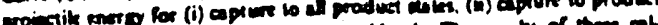

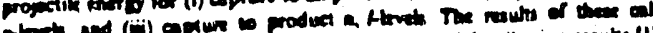

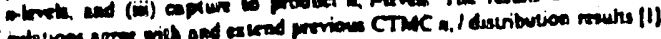




\section{B. ITER NEUTRAL BEAM CURRENT DRIVE}

We have completed a series of cross section calculations central to the determination of a neutral beam penetration in an ITER plasma for assessing the feasibility of current drive. For the dense, high density ITER plasma, a $D^{\circ}$ beam energy of $1 \mathrm{MeV} / \mathrm{u}$ to $2 \mathrm{MeV} / \mathrm{u}$ is anticipated. However, the ionization efficiency of $\mathrm{H}^{\circ}$ by the plasma from not only onestep impact ionization

$$
\mathrm{D}^{\mathrm{O}}+\mathrm{Aq}^{+} \rightarrow \mathrm{D}^{+}+\mathrm{Aq}^{+}+\mathrm{e}^{-},
$$

but via multi-step ionization

$$
D^{0}+\mathrm{Aq}^{+} \rightarrow \mathrm{D}(\mathrm{n})+\mathrm{Aq}^{+}
$$

followed by

$$
D(n)+A q^{+} \rightarrow D\left(n^{\prime}\right)+A q^{+}
$$

or

$$
\mathrm{D}\left(\mathrm{n}^{\prime}\right)+\mathrm{Aq}^{+} \rightarrow \mathrm{D}^{+}+\mathrm{Aq+}+\mathrm{e}^{-}
$$

had to be determined for the main plasma constituents of $\mathrm{A}^{++}=\mathrm{H}^{+}, \mathrm{He}^{++}, \mathrm{C}^{6+}, \mathrm{O}^{8+}$, and $\mathrm{Fe}^{26+}$.

We used a combination of classical-trajectory Monte Carlo, symmetric Eikonal, and atomic orbital coupled-state calculations to provide reliable cross sections for the above reactions. This work, which combines our expertise with that of Wolfgang Fritsch at the Hahn Meitner Institute in Berlin, provides the cross sections over the energy range of $0.25 \mathrm{MeV} / \mathrm{u}$ to $2 \mathrm{MeV} / \mathrm{u}$. The paper describing the theoretical methods and the results has just recently been accepted for publication by Physical Review $A$. 
A modeling study of the ITER neutral beam penetration

has been published in Nuclear Instruments and Methods. In

this paper the magnetic field ionization of the highly

excited deuterium components has been considered along with

the "ladder walking" collisional ionization processes

described above.

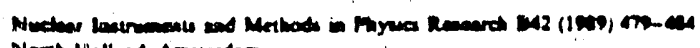

Norib- Helleved. Ameterden

PENETRATON OF AN ENERGETIC D' BEAM INTO AN ETR PLASMA •

J.W. STEARNS. A.S. SCHLACHTER and W.S. COOPER

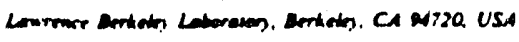

R.E. OLSON

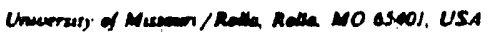

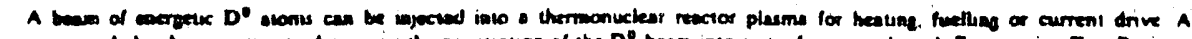

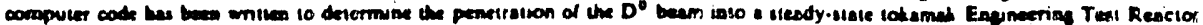

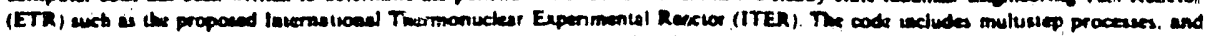

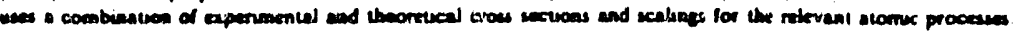

\title{
C. H $^{-}$BEAM BTUDIES
}

We joined in an experimental-theoretical effort to

determine the cross sections for electron removal from $\mathrm{H}^{-}$by collisions with singly and multiply-charged ions

$$
\mathrm{H}^{-}+\mathrm{Aq}^{+} \rightarrow \mathrm{H}^{\circ}+\mathrm{Aq}^{+}+\mathrm{e}^{-}
$$

for charge states $q=1$ to $q=8$. The original work covered the energy range from $3 \mathrm{kev} / \mathrm{u}$ to $100 \mathrm{keV} / \mathrm{u}$, but now it has been extended to $500 \mathrm{kev} / \mathrm{u}$.

One of the motivations behind this study was to assess the feasibility of using a plasma neutralizer for $\mathrm{H}^{-}$to increase the neutral $\mathrm{H}^{\circ}$ yield from about $55 \%$ for gas strippers to around $85 \%$ for a plasma stripper. 
Our modeling studies do indeed indicate an $85 \%$ yield for the plasma stripper when using the newly measured and calculated cross sections. However, even at $500 \mathrm{kev} / \mathrm{u}$, the stripping cross sections do not scale as $q^{2}$, but rather as $q^{1.3}$, which translates to an unacceptable path length for a plasma stripper configuration. This work was published in

\section{Europhysics Letters.}

EUROArmacs LETTERS

1 July 1989

Burophys. Latt., (5), pp. $433-439$ (1909)

Single- and Double-Electron Removal from $\mathrm{H}^{-}$in Energetic Collisions with Multiply-Charged Argon Ions.

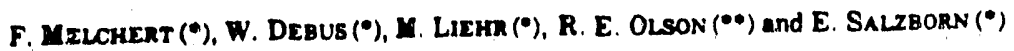
(") Inotitul fur Kemphysik, Strahlenzentrum, Universilat Giessen

D-6soo Gieseen, Wast Germany

(*) Physica Dopartment, Uniurrity of Missouri Rolla - MO sss01, U.S.A.

(received 22 March 1989; accepted 20 April 1969)

PACS 24.70 - Churge tranafor.

PACS C2.20H - ALbic, moleculur, ion and beary particle collisions.

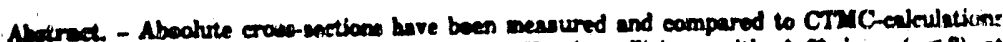

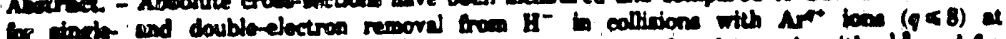

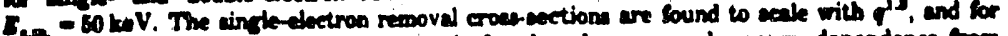

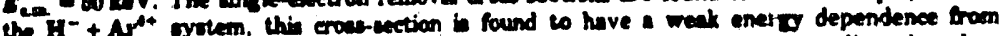

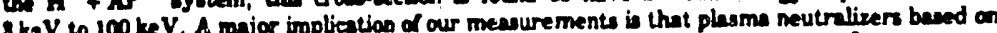

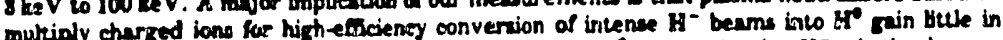
mukiply charged ions lat highte to the trapplicability of $q^{2}$-ecaling in the $\mathrm{H}^{-}$cingle-election removal eroess-mection.

\section{HE ATOY AND ION ANGULAR BCATTERING}

We have collaborated with Tony Donne at the FOM Institute for Plasma Physics in Amsterdam on the interpretation of using a neutral He atom probe for measuring ion temperatures in a fusion plasma. We provided the angular scattering cross sections and neutral atom survival probabilities required to analyze data taken at JT-60 and make predictions for applications to future tokamak fusion devices. Within this study, we investigated single and 
double ionization and charge exchange in small impact parameter collisions with $\mathrm{H}^{+}, \mathrm{C}^{6+}$, and $\mathrm{O}^{8+}$ ions in the range from $25 \mathrm{keV} / \mathrm{u}$ to $100 \mathrm{keV} / \mathrm{u}$.' Good agreement was realized for the comparison of this technique to other methods for giving the impurity ion concentration in the JT-60 plasma, indicating the feasibility of future applications. At present, we are continuing to provide cross sections for this modeling effort.

In order to have confidence in the cross sections we are providing for the fusion effort, we continue to benchmark our calculations against fundamental atomic physics data. In the three studies listed below, we have been able to successfully predict the complete three-body transverse momentum spectra for ionizing collisions between $\mathrm{H}^{+}$and $\mathrm{He}$. This bodes well for our collaboration with Donne in providing the atomic physics data cur neutral beam ion probes. Also, we have demonstrated that our theoretical methods predict the angular, and energy, profiles of the electrons ejected in collisions between $\mathrm{H}^{+}, \mathrm{He}^{++}$and $\mathrm{He}$ in the energy range of 50 $\mathrm{kev} / \mathrm{u}$ to $500 \mathrm{kev} / \mathrm{u}$.

The role of amall-impact-parameter eloctron-lose processes on lon temperafures meseured by actlve-beam plasmi dlagnostices

A. J.H. Dorm

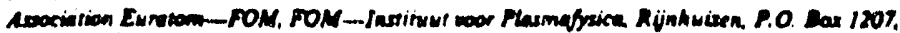
3430 DE Ninuserein Tho Netherband

F. J. How

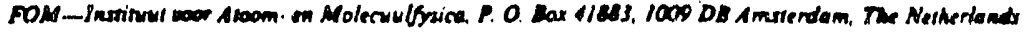

A. E. Otron

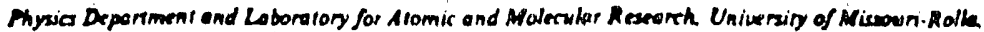
Althe Miowowi areol

(Received 19 May 1988; accepled for publication 6 Seplember 1988)

The shape of the energy distribution of ecaftered particles in an ective-beam scatterias experiment can be infuenced considerably by the presence of bighly charged impurity ican in the plama. In this wart if is shown ineoretically that multiply charged impurities bave anty unall efoct on the ecattering distribution al bow energies ( < $10 \mathrm{keV} / \mathrm{amu}$ ) and at high energies ( > $79 \mathrm{keV} / \mathrm{umv}$ ). Tue etiect of the impurities is the most pronounced for enerpien clave 10 so LeV/amu. The ratuls of the ca'sulations are in good agreement with experimental results a various enerpice Eximpoistion to higher beam energies leads to the conclusion that the active beam scartering diagnostics can be applied also in fulure husion devices for measuring the boul ion temperature. 


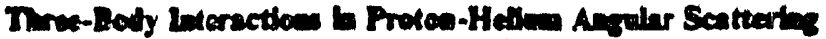

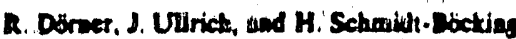

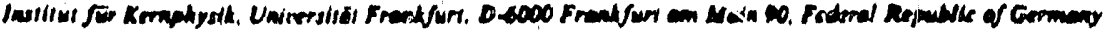

R. E Oron

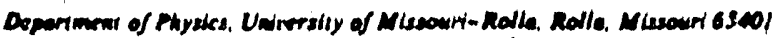

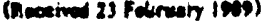

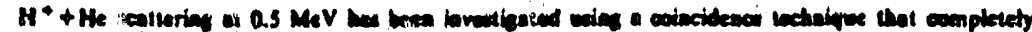

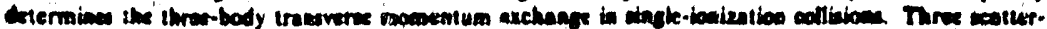

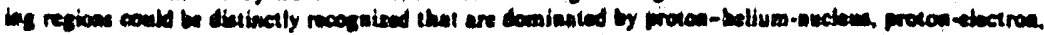

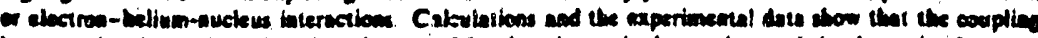

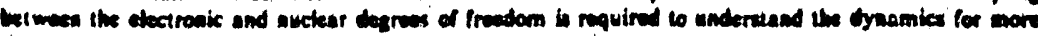
than or of the bonising collibion.

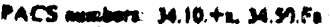

Single- and double-tonlzation cross sections for angular scatteriag of fast protons by bellum

\section{R. E. Oteon}

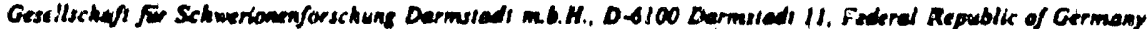

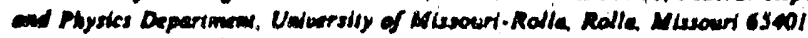

J. Ullich, R. Dörnex, and H. Schmidt-Böcking

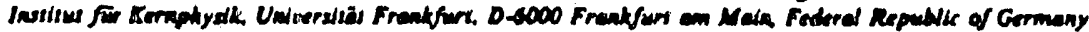

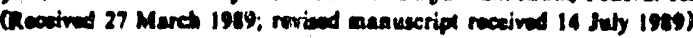

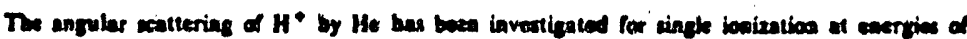

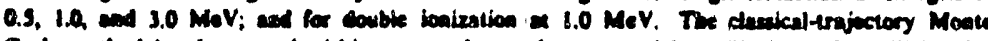

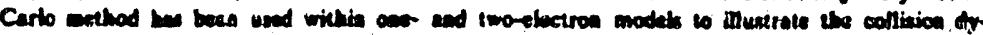

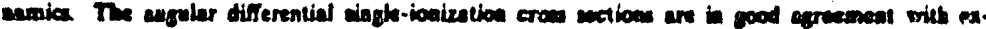

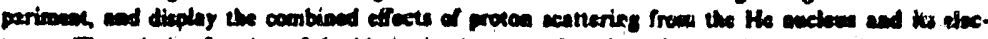

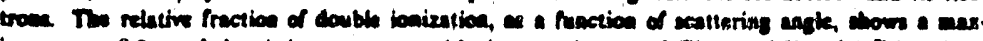

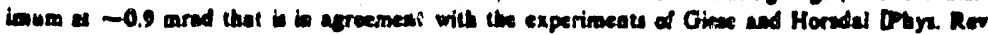

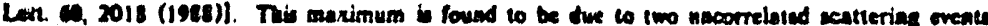

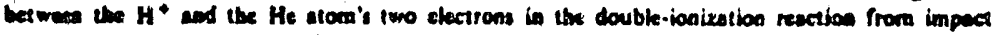
paraneter diferent than that for the single-ionization reaction.

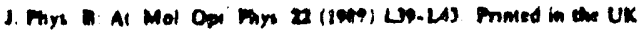

\section{UTTER TO THE EDTrO}

Study of the dependence of the electron emission spectra on the prolectile change in $\mathrm{H}^{*}, \mathrm{He}^{2+}+\mathrm{He}$ collisions

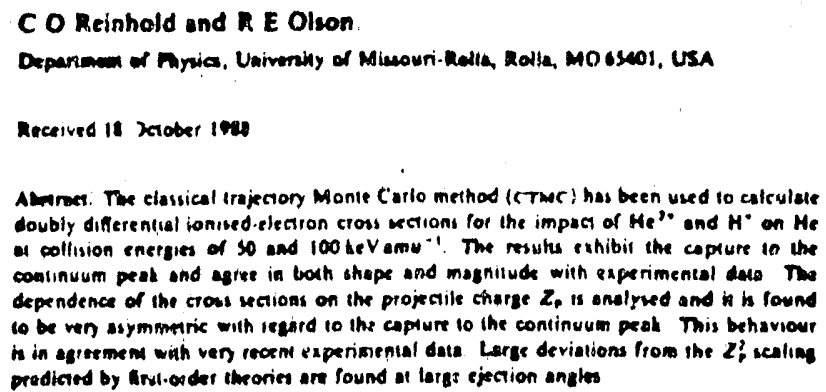

Atannes. The classical trajectory Monte Curlo method (CTMr) has been used to calculate doubly differential ionised electron cross ketions fot the impacy of He" and $\mathrm{H}^{\circ}$ on $\mathrm{He}$

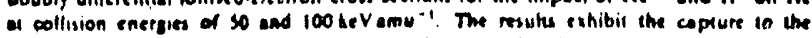

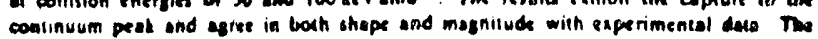

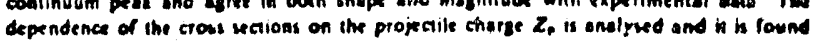
to be ven asymmerric with lesard 10 the capture 10 the continuum peak Mis betaviour

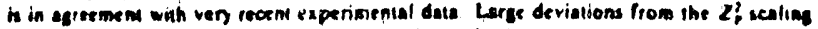

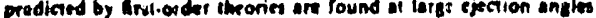




\section{DEVELOPMENTE IN DESCRIBING MANY-ELECTRON TARGETS}

One of our goals over the last several years has been to develop theoretical methods applicable to partially-stripped ions like $\mathrm{Fe}^{10+}$ and many-electron targets such as $\mathrm{He}$ and $\mathrm{H}_{2}$. Accurate methods are increasingly needed for our cross section studies for fusion modeling efforts.

Along these lines, we have finally successfully developed a model potential method that accurately incorporates the screening of the nucleus by the other $\mathrm{N}-1$ electrons in our calculations. Basic to this development was the tion of a momentum distribution description similar to the microcanonical distribution we use for hydrogenic targets in our classical-trajectory Monte Carlo calculations. The procedure uses Thomas-Fermi-Dirac tabulations of the atomic, or ionic, species and now allows us to investigate any ion or atom for nuclear charges from $z=1$ (hydrogen) to $z=54$ (xenon). To investigate the sensitivity of the method, we have performed calculations for not only $\mathrm{H}^{+}$, but also $\mathrm{e}^{-}$and their anti-particles, on He and $\mathrm{Kr}$. Existing experimental data verified our procedure.

Along these same lines, we have developed a classical phase-space model for $\mathrm{H}_{2}$. This method has been successfully applied to electron capture and ionization in collisions with ions in charge states $q=1$ to $q=10$ at energies from $25 \mathrm{keV} / \mathrm{u}$ to $500 \mathrm{kev} / \mathrm{u}$. The calculations clearly show the nonequivalence of the cross sections for $\mathrm{H}_{2}$ with those of doubled $\mathrm{H}^{\circ}$ 
values. Molecular orientation effects were also investigated

\title{
to further probe the collision dynamics.
}

MHYSICAL REVIEW A

\author{
VOLUME \&, NUMBEN?
}

OCTODER 1, 1 N49

Electron remoral from molecular bydrogeb by fully stripped lons at intermediate energies

L. Meng, C. O. Reinhold, and R. E. Olson

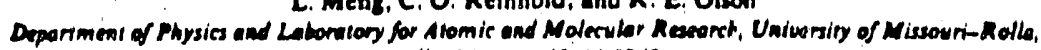 \\ Rolla, Miscowri ossol-ardo \\ Qneceived 19 May 1969 )
}

A classical phase-apace model of the hydrosen molecule is presented and applied to the study of the electron-capture and ionization procesues in collisions of fully uripped lons with $\mathrm{H}_{2}$ ot inter. mediate iapact energies and charge thates from 1 to 10. The model is based on the independenl. clectron and the impact-parameter approximations. The clectron-impinging ion and electroa-urget-nuclei ibleractions ure exactly taken isto account. The iateraction between the efece. troms is epproximnted by noclel polentiab. The calculated coral crous cections for production of free electron and capture of oos electron are in good asreement with diferent experimental dats. The ratio between itre cupture croas eections from molecular and atomis bydrosen tergets is abo an. alyzed and compared with available empirical calings. It is found that this ratio varies from a value less than I if tow impacl energy to a at higher energies The reasons for these differences are discussed. A comparison is made between the sapture cross sections for diferent orientations of the bydrogen molecule.

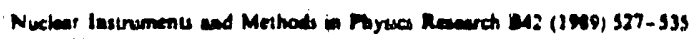
North. Hollesed. Amierden

\section{DINAMICS OF MATTER-, ANTIMATTER-ATOM COULSIONS}

\section{D.R. SCHULTZ, C.O. REINHOLD and R.E. OLSON}

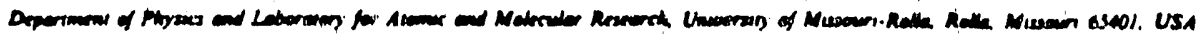

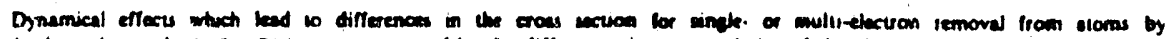

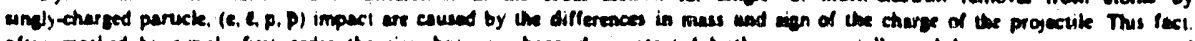

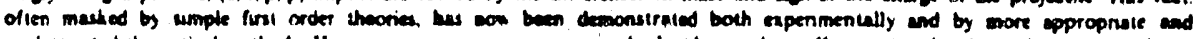

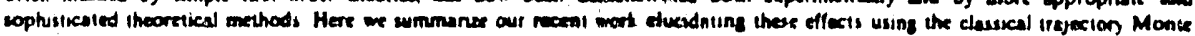

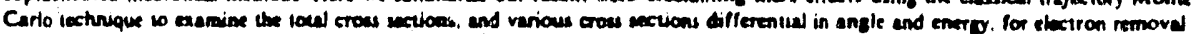

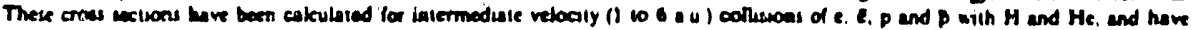
ben comparud with ocher incoretical resulis and expenmenial smesuremeats

Large-angle scaftering in positron-helium and positron-krypton collisioas

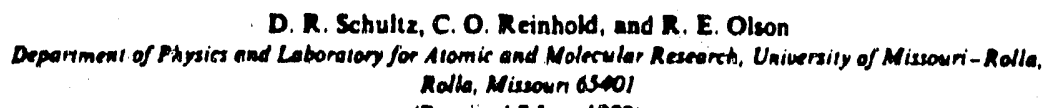

(Received 7 Junc 10s9)

We lave calculated differential cross ecetions as a function of the projectile scaltering angle for ponitron-melium and positron-krypton collisions using the classical-trajectory Monte Carb tecl. nique. These intermediate-velocity sollisions have been simulated by various independentelectron and nekectron models, uaine both ecreened Coulomb and quantum model potentiabs to approximate the effects of electron-electron interactions. These severul models all indicate that scattering of pos Itrons 10 large angles in ionizing collisions persists 10 high impact velocities. In a previous wort [Phys Rev. A 3s, 1006 (1988)] we proposed that the recent experiments that have measured the tosal cross wection for positronium formation could be affected by the lass of positron flux due to in. complete confinemeat after large-angle scattering Here, utilizing these newly calculated differential-scatrering cross sectiom, adjustments are computed that accouni for the difference be. tween the experimental and theoretical behavion of the positranium formation cross cection. Fur. ther, it is demoristrated that brce-angle scalleting in the elastic channel is important for one of the experiments. We have alzo computed total cross sections for conization and charue iransfer for cot. liwons of protons with trypton. Good agreement is obtained in these cases with experimental mes. surements. 


\section{F. ALPEA PARTICLE DIAGNOSTICB}

A major fraction of our effort was spent collaborating with Ray Fisher and Art Howald at General Atomics on carbon pellet alpha particle diagnostics. The basic idea behind the use of the atlation cloud from a carbon pellet is to provide a medium for double electron capture for the alpha particle born at $3.5 \mathrm{MeV}$ by the $\mathrm{D}-\mathrm{T}$ reaction via the double charge exchange reaction:

$$
\mathrm{He}^{++}+\mathrm{Cq}^{+} \rightarrow \mathrm{He}^{\mathrm{O}}+\ldots
$$

Such an idea is intriguing in that electron capture proceeds most readily when there is a velocity match between the projectile velocity and the captured electrons. For carbon, the inherent velocity of the $\mathrm{k}$-shell electrons closely matches that of a $3.5 \mathrm{MeV} \mathrm{He}{ }^{++}$. Thus, it is not necessary to accelerate a neutral beam such as $\mathrm{Li}^{\circ}$ to $6 \mathrm{MeV}$, a costly proposition, when the atomic properties of the target can be used.

originally, the carbon pellet scheme looked extremely promising, as the electron capture primarily proceeds from the $\mathrm{K}$-shell of the carbon, so that partially-stripped $\mathrm{c}^{q+}$ in the ablation cloud with $q=0$ to $q=4$ would all yield the same result.

our involvement in the project is to provide the cross sections necessary to accurately estimate the neutralized fraction of $\mathrm{He}^{\circ}$ produced when an $\mathrm{He}^{++}$traverses a carbon pellet plasma. The needed cross sections are not only the 

double electron capture values for the reaction given above, but also the sequential one-electron capture cross sections for the reactions

$$
\begin{aligned}
& \mathrm{He}^{++}+\mathrm{Cq}^{+} \rightarrow \mathrm{He}^{+}+\ldots \\
& \mathrm{He}^{+}+\mathrm{Cq}^{+} \rightarrow \mathrm{He}^{\mathrm{O}}+\ldots
\end{aligned}
$$

and the competing ionization cross sections for the $\mathrm{He}^{+}$and He? on the carbon plasma constj.tuents. It was also necessary to calculate the $\mathrm{K}$-shell capture relative to $\mathrm{L}$ - and $\mathrm{higher}$ shells in order to predict the equilibrium fractions.

We used the classical-trajectory Monte Carlo method with the newly developed model potentials described in the previous section to estimate the ionization cross sections. Then an atomic orbital calculation was used to obtain the single and double electron $\mathrm{K}$ - to $\mathrm{K}$-shell transfer. These values were then used as input by Art Howald at GA to compute the neutral equilibrium fraction which was compared to Japanese $\mathrm{CH}_{4}$ gas target data. Good agreement was found at $3.5 \mathrm{MeV}$, but serious differences occurred at lower energies.

We then reassessed our assumption of only $\mathrm{K}$ - to $\mathrm{K}$-shell capture and provided another series of calculated cross sections for $\mathrm{L}$ - to $\mathrm{K}$-shell capture from carbon to the alpha particle. At first light, this inclusion appears to remove the discrepancy. However, we will only be able to quantitatively confirm, or reject, our recent insight into the colIision mechanisms when Art returns from Japan and includes our new values in his equilibrium code. 
If this modeling study is successful, we will have generated the atomic physics data base needed for the carbon pellet diagnostic. The uncertainty for the project, however, is that it may now be necessary to know precisely the charge state distribution of the carbon ablation cloud in order to predict the Heo neutral yield.

Along these same lines, we have been concerned abouc the energy a josition (i.e., stopping power) for the alpha particle in the carbon plasma. Original estimates of the slowing down of the alpha by the carbon plasma diagnostic were based on solid targets, not a plasma with long-range coulomb interactions. To investigate this problem more fully, we have developed the capability to calculate stopping powers for ions in gases and solids at MeV energies. These calculations have been benchmarked against existing data and appear appropriate for a future application to the carbon pellet problem.

ENERGY DEPOSITION OY EMERGETIC MEAWY-IONS IN MATTEA

$$
\text { a. E. Otion }
$$

Gesellachen för Schwerionemorachung (GSI), 0-6100 Darmsted 11. FRG and

Physics Departmen, University of Missourtiolla, Rollo, MO GSA1, USA

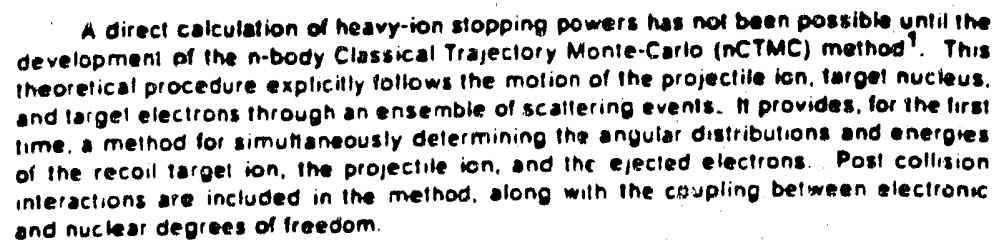

and nuclear degrees of Iraedom. 

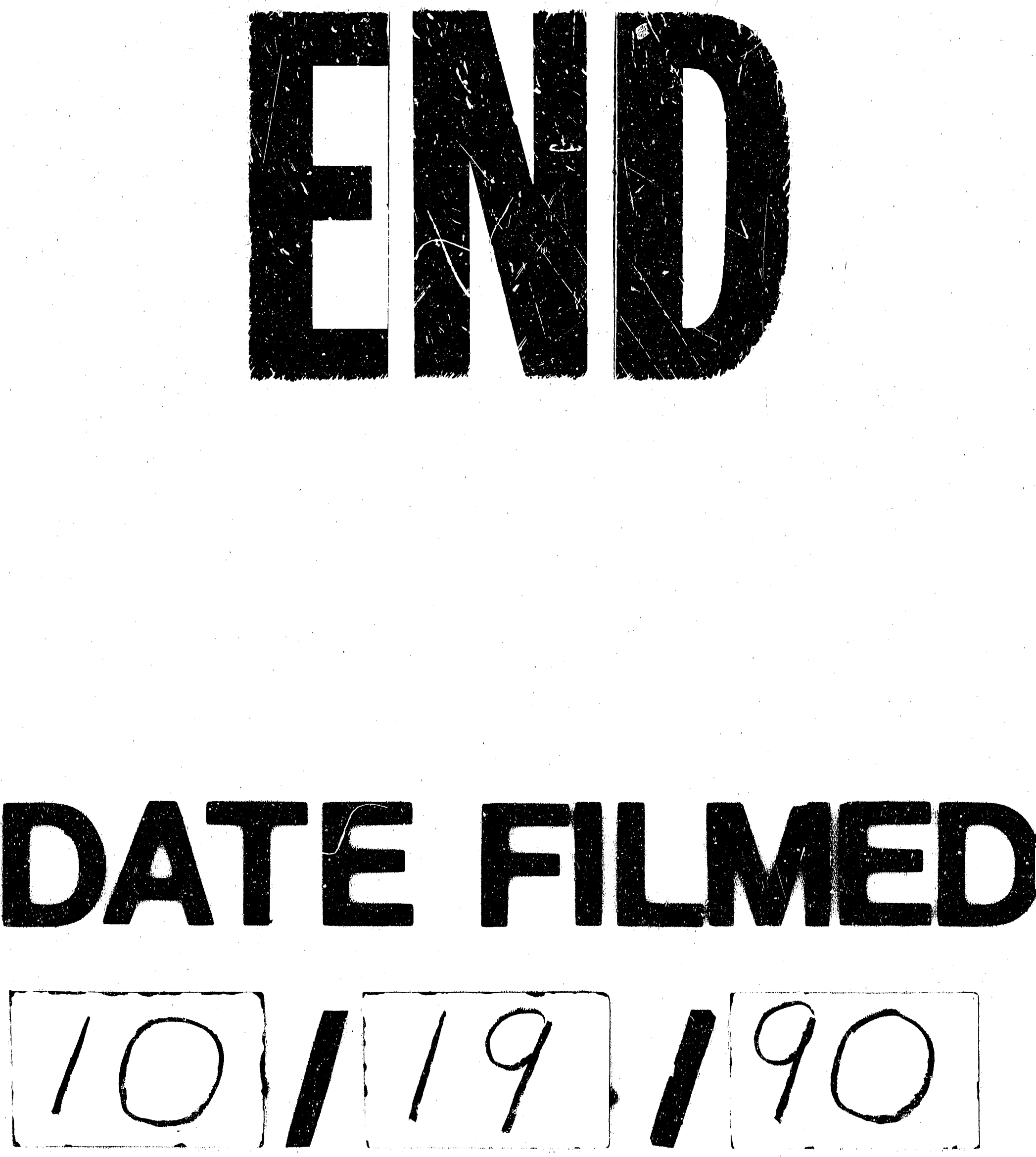
